This document is published in:

J.M. Mateos-Pérez, Manuel Desco, Michael W. Dae, Carmen García-Villalba, Lorena Cussóa, Juan J. Vaquero, Automatic TAC extraction from dynamic cardiac PET imaging using iterative correlation from a population template, Computer Methods and Programs in Biomedicine (2013), 111(2), 308-314. DOI: http://dx.doi.org/10.1016/i.cmpb.2013.04.010

(C) 2013 Elsevier Ireland Ltd. All rights reserved 


\title{
Automatic TAC extraction from dynamic cardiac PET imaging using iterative correlation from a population template
}

\author{
José M. Mateos-Pérez ${ }^{a, b, *}$, Manuel Desco ${ }^{b, c}$, Michael W. Dae $^{d}$, Carmen García-Villalba ${ }^{b}$, \\ Lorena Cussóa ${ }^{a, b}$, Juan J. Vaquero ${ }^{c}$ \\ a CIBERSAM, Hospital General Universitario Gregorio Marañón, Madrid, Spain \\ $\mathrm{b}$ Instituto de Investigación Sanitaria Gregorio Marañón, Madrid, Spain \\ c Departamento de Bioingeniería e Ingeniería Aeroespacial, Universidad Carlos III de Madrid, Madrid, Spain \\ d Department of Radiology \& Biomedical Imaging, University of California, San Francisco, USA
}

Keywords:

Pet

Cardiac imaging

Automatic segmentation

Kinetic modeling

\begin{abstract}
A B S T R A C T
This work describes a new iterative method for extracting time-activity curves (TAC) from dynamic imaging studies using a priori information from generic models obtained from TAC templates. Analytical expressions of the TAC templates were derived from TACs obtained by manual segmentation of three ${ }^{13} \mathrm{NH}_{3}$ pig studies (gold standard). An iterative method for extracting both ventricular and myocardial TACs using models of the curves obtained as an initial template was then implemented and tested. These TACs were extracted from masked and unmasked images; masking was applied to remove the lungs and surrounding nonrelevant structures. The resulting TACs were then compared with TACs obtained manually; the results of kinetic analysis were also compared. Extraction of TACs for each region was sensitive to the presence of other organs (e.g., lungs) in the image. Masking the volume of interest noticeably reduces error. The proposed method yields good results in terms of TAC definition and kinetic parameter estimation, even when the initial TAC templates do not accurately match specific tracer kinetics.
\end{abstract}

\section{Introduction}

Quantitative kinetic analysis of dynamic cardiac PET data provides unique information that can improve discrimination between different states of myocardial tissue $[1,2]$. Conventional estimation of kinetic parameters based on compartmental models requires an accurate assessment of arterial blood input function. An image-derived input function removes the need for invasive blood sampling $[1,3,4]$.
One commonly used method involves manually defining a region of interest (ROI) [1,5-7]. However, this can be a slow and challenging process, especially in noisy image series, where considerable care is needed to manually select the appropriate areas. Furthermore, the manual ROI segmentation process is operator-dependent and has low reproducibility [8]. Therefore, a simple but reliable procedure to automatically extract the time-activity curve (TAC) would be advantageous, and several automatic or semi-automatic techniques have been proposed to address this issue. If these tools are to be applied in clinical

\footnotetext{
* Corresponding author at: Unidad de Medicina Experimental, Hospital General Universitario Gregorio Marañón, C/Doctor Esquerdo 46, 28007 Madrid, Spain. Tel.: +34 91586 6678; fax: +34 914265108.

E-mail address: jmmateos@mce.hggm.es (J.M. Mateos-Pérez).
} 
practice, they should be user-friendly and provide consistent and reliable results.

Factor analysis of dynamic structures has removed the need for manual segmentation [9-11]. Although this approach is used extensively for semi-automatic TAC extraction, its main drawback is the non-uniqueness of the solution, which in practice is observed as spatial overlap between factor images from different regions [2]. Several modifications to the original algorithm have been proposed to correct this problem $[12,13]$ without using a priori information, mainly by imposing additional restrictions on those factor coefficients considered valid by the algorithm and thus penalizing overlap between regions. However, these modifications minimize but do not completely eliminate spatial overlap.

Other semi-automatic methods, such as covariance images calculated from an initial manual ROI, have been published [14], although they require manual intervention from the user.

Several proposed solutions involve clustering techniques [8,15-20]. For algorithms such as the classic $k$-means [21], it is important to know the number of clusters a priori to obtain an optimum result $[8,16]$; however, this information is often unknown and it is not easy to compare results obtained with different values of $k$ [16]. Furthermore, $k$-means uses a random initialization process that may cause it to fall into a local minimum.

Since the dynamics of the tracer used are often well known, the solution to this problem could be simplified in order to extract the TACs using a previous kinetic model and a similarity metric. Prior modeling of the approximate solution has successfully applied in other applications [22].

We present proof of concept for an iterative clustering method that makes it possible to automatically obtain the image-derived blood input function and myocardial TACs from dynamic cardiac PET studies. We apply a priori models of tracer kinetic behavior in different regions or tissues obtained from a population sample and not from the study being segmented; this approach resembles $k$-means but requires considerable $a$ priori knowledge of the problem. No manual intervention from the operator is necessary. In addition, the technique is fast and provides robust results based on the original data (which are therefore physiologically interpretable).

\section{Materials and methods}

\subsection{Method definition}

\subsubsection{Model}

PET studies using ${ }^{13} \mathrm{~N}$-ammonia $(740 \mathrm{MBq})$ in healthy pigs were obtained at rest and under stress conditions. Images were acquired using a matrix size of $128 \times 128 \times 47$ pixels, with a voxel size of $2.34 \mathrm{~mm} \times 2.34 \mathrm{~mm} \times 3.27 \mathrm{~mm}$. Twentyfive dynamic frames were acquired over a total of 900 seconds (18 $\times 5$-s frames, $2 \times 15$-s frames, $3 \times 60$-s frames, and $2 \times 300$ $\mathrm{s}$ frames). Tracer was injected as a bolus using an automatic device. All the studies were acquired using a Discovery STE scanner (General Electric Healthcare, Connecticut, USA) in 2D mode and reconstructed using a filtered back-projection algorithm at $2 \mathrm{~mm}$ /pixel with a 4.8 - $\mathrm{mm}$ cutoff and no post-filtering or scatter correction with convolution subtraction. Images were masked to include only the relevant tissues.

The different regions (left ventricle (LV), right ventricle (RV) and myocardium (MYO)) were manually segmented and their mean TACs were obtained. TAC templates corresponding to these three different volumes of interest were generated using function fitting with nonlinear least-squares. Three TAC templates were generated (LV, RV and MYO), one for each pair of studies, in order to use a leave-one-out approach in the validation stage (see Section 2.3). The RV and LV TACs are defined by a gamma function according to the equation

$f(t)=K t^{\alpha} e^{-(t / \beta)}$

The MYO curve was modeled on an exponential plus a constant factor that corrects for late time slope, as follows:

$f(t)=k\left(1-e^{-t / \alpha}\right)+\beta t$

Exact values of $K, \alpha$, and $\beta$ in Eqs. (1.1) and (1.2) depend on the particular set of curves used to define the template; in our case, we defined the LV TAC in the same way as the RV TAC, but with a delayed maximum and slower decay, whereas the MYO reached a smooth plateau after an exponential increase.

As these are the first models in the iterative process, their initial formulation does not necessarily describe the final result and it is indeed just an approximation. Furthermore, a gamma function fit does not provide as good a result as triple exponential or other similar functions; nevertheless, the purpose of the initial curves is to have a generic model of tracer behavior that is similar in shape to as many studies as possible. An example of these initial templates can be seen in Fig. 1, along with the manually-derived curves.

\subsubsection{Iterative algorithm for TAC extraction}

The method proposed iteratively updates the TACs corresponding to the three regions, starting with the previously defined templates. The resulting curves are not fitted after each iteration; instead, the average TAC for the region is used. In each iteration, the method calculates three correlation images, one for each region (LV, RV, and MYO). The value of each new voxel in these images is the result of computing the correlation for the TAC of that particular voxel with the corresponding template. The Pearson correlation is used for this calculation as follows:

$\rho_{X, y}=\frac{\operatorname{cov}(X, Y)}{\sigma_{X} \sigma_{Y}}=\frac{E\left[\left(X-\mu_{X}\right)\left(Y-\mu_{Y}\right)\right]}{\sigma_{X} \sigma_{Y}}$

with $X$ and $Y$ being any two TACs, and $\mu$ and $\sigma$ their mean value and standard deviation, respectively. In our case, $X$ will be the TAC from a given voxel and Y the TAC from the template.

Each iteration comprises three steps:

1. The correlation between the TAC measured for each smoothed voxel (3 kernel points, $\{0.25,0.50,0.25\}$ ) and the templates for LV, RV, and MYO are computed. To avoid noisy TACs, the corresponding TAC maximum value must be positive and greater than the absolute value of the TAC minimum (which can be negative due to noise), otherwise 


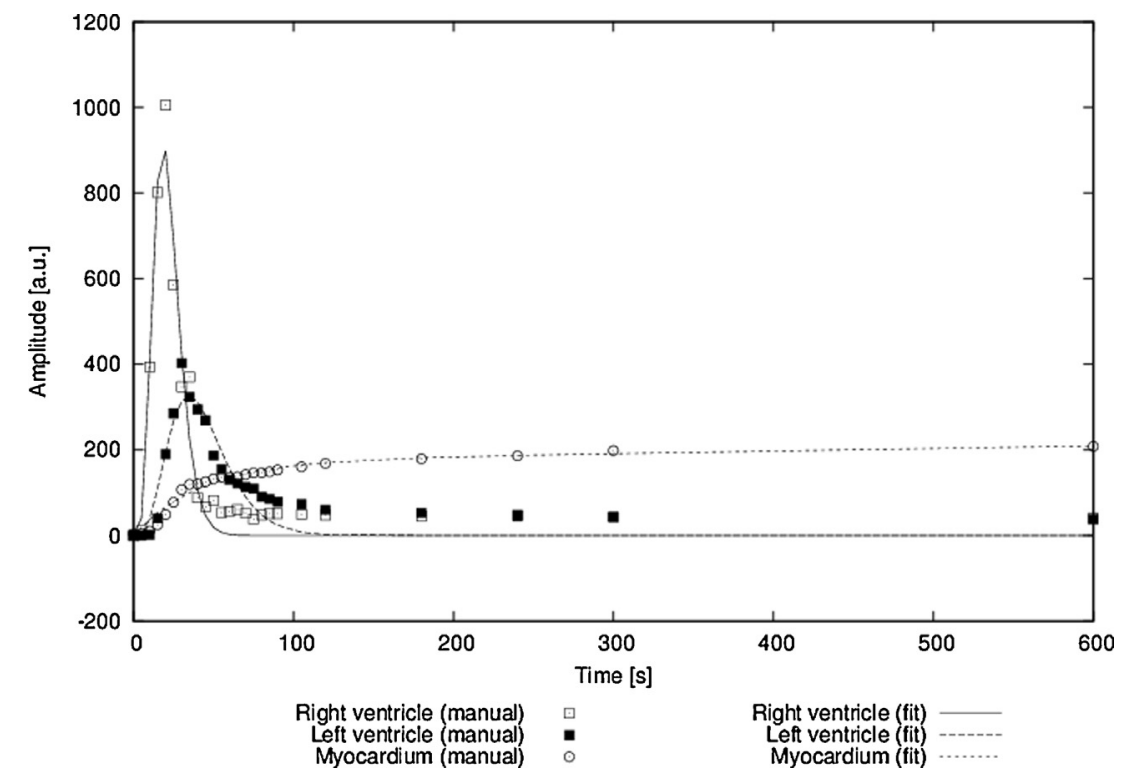

Fig. 1 - TACs extracted manually along with the fit used as the initial template. The data shown here are for one of the leave-one-out cases.

the TAC is skipped. For those voxels that comply with these conditions, the largest of the three correlation values is assigned to the corresponding correlation image (LV, RV, or $\mathrm{MYO}$ ) if it is higher than a predefined correlation threshold and is set at zero in the other two images.

2. Each correlation image is filtered using a $3 \times 3$ median filter to eliminate isolated voxels. The remaining voxels are set at 0 , thus resulting in a binary volume mask.

3. A new TAC for each region is calculated by averaging the TACs of those voxels determined by the masks, and this new TAC is used as the template for the next iteration.

The correlation thresholds used at each iteration are $\{0.2$, $0.3,0.4,0.5,0.6,0.7,0.8,0.9$, and 0.95$\}$ for a total of nine iterations. The rationale for these correlation scores is that the initial model comes from the fitted template and does not precisely represents the specific curves for the problem being solved; therefore, low correlation scores must be used to find the actual study curves that resemble those of the model. Once those curves have been found, spurious data are filtered out by steadily increasing the correlation score used.

After the final iteration, three volumes are obtained, one for each tissue type (LV, RV, MYO). Each volume contains the correlation values for every voxel. To define the final TACs, a two-step selection process is applied. First, in order to eliminate voxels with low correlation values from the final calculation, only those voxels whose correlation value is in the 70th percentile for each specific correlation image are kept. In the following step, the maximum TAC amplitude is computed for all the voxels corresponding to the LV and RV images obtained from the previous filtering. Finally, an average curve is computed from those voxels that have a TAC whose amplitude is above the 50th percentile. This step is only required for calculation of the ventricular cavity TACs and is needed to remove low-amplitude voxels that smooth the final image-derived input function. The resulting images after this last step are also saved and used to visually check the regions from where the TAC was finally extracted. These images are offered only as a reference, as the actual results obtained by this algorithm are the TACs for each region.

The algorithm was developed in Java as a plug-in for the PMOD software package (PMOD Technologies Ltd., Zurich, Switzerland). All tests were carried out on an Intel Core 2 Quad CPU with 4 GB of RAM. ImageJ v1.42 (National Institutes of Health, Bethesda, Maryland, USA) and COLT v1.2.0 libraries (CERN - European Organization for Nuclear Research, Geneva, Switzerland) were used for image processing.

\subsection{Gold standard}

To create a gold standard, all the studies were manually segmented by three specialists. Although the usual gold standard for these measurements is arterial sampling, such data were not available; therefore, the image-derived input functions were used as a surrogate measure [1,3,4]. Furthermore, as this method was developed to extract the input function and tissue curves from image data, a comparison had to be made against the manually obtained TAC.

\subsection{Data analysis}

Due to the small sample size, cross-validation was carried out using a leave-one-out approach: templates were obtained with each pair of studies (study 1 and 2, study 2 and 3, study 1 and 3) and used to segment the remaining study. This is a cross-validation approach useful in studies where the number of cases is small, such as the present one. Mean TACs were generated for each region, the curves were fitted and used to segment the third study. 
Table 1 - Final results (error and correlation score) of the comparison between automatic segmentation and an average manual segmentation curve.

\begin{tabular}{|c|c|c|c|c|c|c|}
\hline \multirow[t]{2}{*}{ Study } & \multicolumn{2}{|c|}{ Left ventricle } & \multicolumn{2}{|c|}{ Right ventricle } & \multicolumn{2}{|c|}{ Myocardium } \\
\hline & Error (\%) & $r$ & Error (\%) & $r$ & Error (\%) & $r$ \\
\hline$\# 1$ & 2.56 & 0.99 & 4.95 & 0.99 & 8.31 & 0.97 \\
\hline \#2 & 6.38 & 0.99 & 5.84 & 0.99 & 6.35 & 0.99 \\
\hline \#3 & 3.10 & 0.99 & 6.95 & 0.98 & 12.82 & 0.97 \\
\hline
\end{tabular}

TACs obtained automatically and manually were compared using the normalized root-mean-square deviation (NRMSD):

$\operatorname{NRMSD}(\%)=\frac{\sqrt{\frac{\Sigma\left(\operatorname{TAC}_{\text {automatic }}(t)-\mathrm{TAC}_{\text {manual }}(t)\right)^{2}}{n}}}{\mathrm{TAC}_{\max }-\mathrm{TAC}_{\min }} \cdot 100$

where $\mathrm{TAC}_{\text {automatic }}$ is the TAC obtained by the algorithm, $\mathrm{TAC}_{\text {manual }}$ is the average TAC for all the manual segmentations for that particular region, $n$ is the total time points acquired, and $\mathrm{TAC}_{\max }$ and $\mathrm{TAC}_{\min }$ are the maximum and minimum values for both TACs.

The Pearson correlation coefficient between manual and automatic results was also computed. To perform a visual evaluation of the final segmentation, static images were created by adding the last five frames of the dynamic images.

Kinetic parameters $\left(K_{1}[\mathrm{ml} / \mathrm{min} / \mathrm{g}]\right.$ and $\left.k_{2}\left[\mathrm{~min}^{-1}\right]\right)$ and spillover from LV into MYO (vLV) were computed using a 1-compartment model [23] implemented in PMOD's kinetic analysis module. All the kinetic parameters are shown as a pair consisting of a value and its standard error. This standard error is yielded by PMOD's iterative fitting process and is a measurement of the correctness of the kinetic parameter.

\section{Results}

The cross-validation process was completed successfully using a leave-one-out approach, and all the studies were segmented employing less than half minute computing time $(17.86 \pm 2.28 \mathrm{~s} ;$ mean \pm standard deviation). The TACs obtained automatically very closely resembled those obtained via manual segmentation (Table 1), both in shape and in amplitude ( $r \geq 0.97$ for all regions).

An example of the resulting visual segmentation can be seen in Fig. 2. It is important to note that visual segmentation is not the main outcome of this algorithm and is produced only for verification purposes. The TACs obtained for this segmentation are shown in Fig. 3.
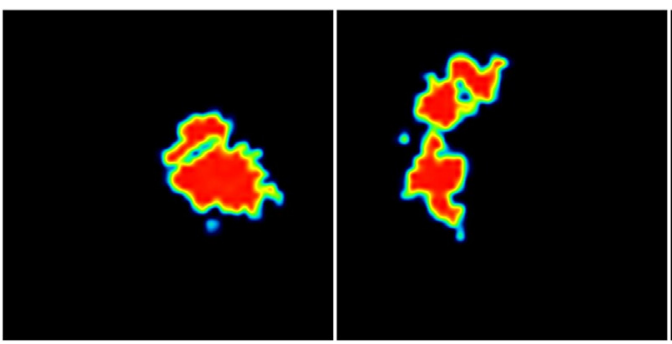

The results of the kinetic analysis (Table 2) show that both the manually and automatically obtained curves yield similar results for myocardial blood flow $\left(K_{1}\right)$, with the automatic TACs offering the smallest errors (COV) in the iterative process in almost all cases and the smallest spillover (vLV) from LV into MYO.

\section{Discussion}

The method proposed enabled us to successfully extract TACs for LV, RV, and MYO in all the studies using a leave-one-out approach. The TACs extracted were then used as input in a kinetic analysis module, and the results show excellent agreement with those of the manually obtained TACs.

This method has several major differences with the classic $k$-means algorithm. First, the initial TACs are not obtained by selecting random voxels on the image, but are instead generated from a population sample, given that the kinetics of the tracer are known. Consequently, the results for the algorithm presented are deterministic, that is, they are always the same when applied to the same study, whereas $k$-means may fall into local minima [16]. In addition, there is no need to find the optimal value of regions to be segmented $[8,19]$, as the final result will yield as many TACs as templates used. Although only three templates were used in the present study, the algorithm could be used in principle with any number of regions.

No overlap was observed between the different regions; this is an improvement over factor analysis, in which overlap can be minimized in the factor images, but not completely removed [2,12]. Note that we are referring to image overlapping (that is, a voxel that simultaneously belongs to more than one region); spill-over and partial volume effects may still be present.

Despite the small sample size, the models accomplished their task and were used to locate similar curves inside the actual study. These curves were then refined; given that the

Fig. 2 - Visual results of the segmentation for one of the studies. From left to right: left ventricle, right ventricle, myocardium, and a reference image created by adding the last 5 frames of the study. 


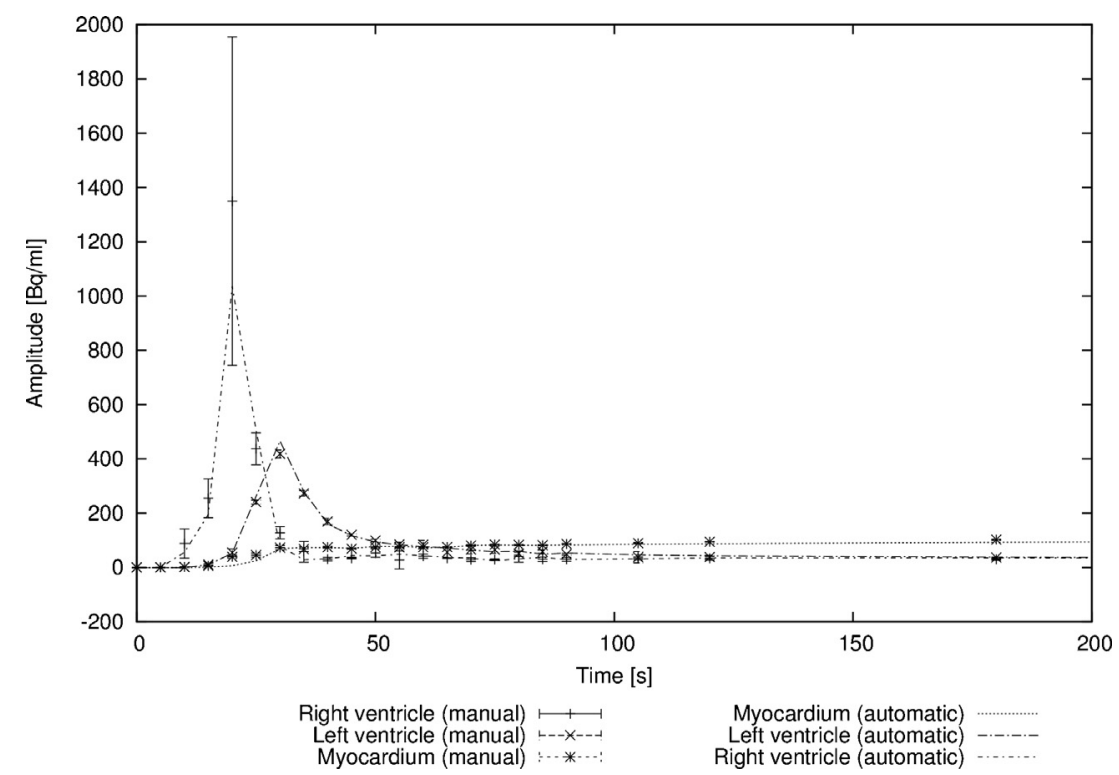

Fig. 3 - Time-activity curves for the study shown in Fig. 2. The time axis has been truncated to 200 seconds to emphasize the values for the TACs during the first moments of the acquisition. The peak value for the automatic segmentation is noticeably smaller than the mean manual value due to one of the operators drawing a ROI that yielded abnormally high values.

correlation score is increased every time, the number of pixels that is allowed inside each region diminishes with each iteration. In the graphical segmentations, the holes visible in some regions (e.g., RV in Fig. 2) are a consequence of the high correlation scores used in the last iterations of the algorithms. If the shape of a given TAC does not correlate highly with the one being used as the tissue TAC, that voxel will be skipped. As the objective of the segmentation process is to extract accurate TACs to be used as input in kinetic analysis procedures, imperfections in the visual result (which serves merely as a guide to the operator) are not relevant.

Measured myocardial blood flow is similar with both methods, although automatic segmentation provides a tissue TAC that is much less affected by spillover from LV, as shown in the kinetic analysis results via the vLV variable. This spillover may be responsible for the low $k_{2}$ values obtained in the manual segmentations. Therefore, the algorithm is successful in separating different TACs and can eliminate voxels that would be included in MYO by a human operator but are affected by spillover from the left ventricle and should be left out of that ROI.

Use of a sample population for the initial population is problematic. On the one hand, in order to automate this step in a clinical environment, a library with standard models can be defined for the most commonly used tracers. On the other hand, given that the algorithm needs a priori information, pathological tissue might not be correctly segmented if its kinetics is too different from that used to build the models. This may also be the case when the template has been generated using a different injection type. These problems could be solved by generating templates for different pathologies and injection types, although further testing should be carried out in this regard. Furthermore, failure to perform segmentation correctly may signal pathology when a healthy model has been

Table 2 - Kinetic analysis parameters. "COV" represents the coefficients of variation for that parameter after kinetic analysis modeling.

\begin{tabular}{|c|c|c|c|c|c|c|}
\hline Study & $K_{1}$ & $\mathrm{COV}$ & $k_{2}$ & $\mathrm{COV}$ & vLV & $\mathrm{COV}$ \\
\hline \#1, op 1 & 0.622057 & 5.49 & 0.118421 & 45.41 & 0.115477 & 14.07 \\
\hline$\# 1$, op 2 & 0.628333 & 3.66 & 0.106127 & 33.58 & 0.140943 & 7.57 \\
\hline \#1, op3 & 0.651833 & 6.37 & 0.051074 & 116.43 & 0.127073 & 15.56 \\
\hline$\# 1$, auto & 0.656951 & 2.17 & 0.2163 & 10.81 & 0.08639 & 7.06 \\
\hline \#2, op1 & 1.094091 & 2.75 & 0.199012 & 15.48 & 0.164741 & 9.61 \\
\hline$\# 2$, op2 & 0.961911 & 2.44 & 0.141246 & 18.2 & 0.158114 & 8.06 \\
\hline \#2, ор3 & 1.17784 & 3.76 & 0.149479 & 27.28 & 0.167962 & 14.15 \\
\hline \#2, auto & 0.918549 & 1.88 & 0.206826 & 10.02 & 0.120561 & 7.17 \\
\hline \#3, op1 & 0.489181 & 6.0 & 0.100358 & 56.61 & 0.15453 & 9.05 \\
\hline$\# 3$, op2 & 0.480133 & 3.04 & 0.101073 & 28.67 & 0.136645 & 5.17 \\
\hline \#3, op3 & 0.46374 & 6.17 & 0.05567 & 101.18 & 0.149009 & 9.26 \\
\hline \#3, auto & 0.477204 & 2.45 & 0.250987 & 10.66 & 0.087988 & 5.81 \\
\hline
\end{tabular}


used. In any case, pathology is less of an issue in the case of input function extraction, as its activity should be less influenced by cardiac pathology than surrounding tissues and is, in general, more robust than the myocardium TAC, as reflected by methods such as population-based input functions [24]; this method would therefore provide a good input function extracted from the image data.

\section{Conclusion}

Automatic and fast TAC extraction is feasible using an iterative correlation algorithm with a general initial template resembling a specific tracer. The resulting segmentations provide TACs close to those obtained manually, and kinetic analysis yields similar results. Spillover into MYO is much smaller with the automatic method.

\section{Acknowledgments}

This work is supported by the following grants: RD07/0014/2009, Subprograma RETICS, Ministerio de Ciencia e Innovación. S2009/DPI-1802 (ARTEMIS), Comunidad de Madrid. CEN-20101014, Programa CENIT, CDTI, Ministerio de Ciencia e Innovación. European Commission, EFPIA, INNOVATIVE MEDICINE INITIATIVE (PredDICT-TB project, 115337-1).

\section{R E F E R E N C E S}

[1] E. Croteau, E. Lavallée, S.M. Labbe, L. Hubert, F. Pifferi, J.A. Rousseau, S.C. Cunnane, A.C. Carpentier, R. Lecomte, F. Bénard, Image-derived input function in dynamic human PET/CT: methodology and validation with 11C-acetate and 18F-fluorothioheptadecanoic acid in muscle and 18F-fluorodeoxyglucose in brain, European Journal of Nuclear Medicine and Molecular Imaging 37 (August (8)) (2010) 1539-1550.

[2] J. Kim, P. Herrero, T. Sharp, R. Laforest, D.J. Rowland, Y.-C. Tai, J.S. Lewis, M.J. Welch, Minimally invasive method of determining blood input function from PET images in rodents, Journal of Nuclear Medicine: Official Publication, Society of Nuclear Medicine 47 (February (2)) (2006) 330-336.

[3] I.N. Weinberg, S.C. Huang, E.J. Hoffman, L. Araujo, C. Nienaber, M.G. McKay, M. Dahlbom, H. Schelbert, Validation of PET-acquired input functions for cardiac studies, Journal of Nuclear Medicine 29 (2) (1988) 241-247.

[4] L.-F. de Geus-Oei, E.P. Visser, P.F.M. Krabbe, B.a. van Hoorn, E.B. Koenders, A.T. Willemsen, J. Pruim, F.H.M. Corstens, W.J.G. Oyen, Comparison of image-derived and arterial input functions for estimating the rate of glucose metabolism in therapy-monitoring 18F-FDG PET studies, Journal of Nuclear Medicine: Official Publication, Society of Nuclear Medicine 47 (June (6)) (2006) 945-949.

[5] J.E.M. Mourik, M. Lubberink, A. Schuitemaker, N. Tolboom, B.N.M. van Berckel, A.A. Lammertsma, R. Boellaard, Image-derived input functions for PET brain studies, European Journal of Nuclear Medicine and Molecular Imaging 36 (March (3)) (2009) 463-471.

[6] M.N. Tantawy, T.E. Peterson, Simplified [18F]FDG image-derived input function using the left ventricle, liver, and one venous blood sample, Molecular Imaging: Official
Journal of the Society for Molecular Imaging 9 (April (2)) (2010) 76-86.

[7] A.P. van der Weerdt, L.J. Klein, R. Boellaard, C.A. Visser, F.C. Visser, A.A. Lammertsma, Image-derived input functions for determination of MRGlu in cardiac (18)F-FDG PET scans, Journal of Nuclear Medicine: Official Publication, Society of Nuclear Medicine 42 (November (11)) (2001) 1622-1629.

[8] K.-P. Wong, D. Feng, S.R. Meikle, M.J. Fulham, Segmentation of dynamic PET images using cluster analysis, IEEE Transactions on Nuclear Science 49 (February (1)) (2002) 200-207.

[9] D.C. Barber, The use of principal components in the quantitative analysis of gamma camera dynamic studies, Physics in Medicine and Biology 25 (March (2)) (1980) 283-292.

[10] R. Di Paola, J.P. Bazin, F. Aubry, A. Aurengo, F. Cavailloles, J.Y. Herry, E. Kahn, Handling of dynamic sequences in nuclear medicine, IEEE Transactions on Nuclear Science 29 (4) (1982) 1310-1321.

[11] H.M. Wu, C.K. Hoh, Y. Choi, H.R. Schelbert, R.A. Hawkins, M.E. Phelps, S.C. Huang, Factor analysis for extraction of blood time-activity curves in dynamic FDG-PET studies, Journal of Nuclear Medicine: Official Publication, Society of Nuclear Medicine 36 (September (9)) (1995) 1714-1722.

[12] G. El Fakhri, A. Sitek, B. Guérin, M.F. Kijewski, M.F. Di Carli, S.C. Moore, Quantitative dynamic cardiac 82Rb PET using generalized factor and compartment analyses, Journal of Nuclear Medicine: Official Publication, Society of Nuclear Medicine 46 (August (8)) (2005) 1264-1271.

[13] A. Sitek, G.T. Gullberg, R.H. Huesman, Correction for ambiguous solutions in factor analysis using a penalized least squares objective, IEEE Transactions on Medical Imaging 21 (March (3)) (2002) 216-225.

[14] A.O. Boudraa, J. Champier, M. Djebali, F. Behloul, A. Beghdadi, Analysis of dynamic nuclear cardiac images by covariance function. Computerized medical imaging and graphics, The Official Journal of the Computerized Medical Imaging Society 23 (4) (1999) 181-191.

[15] X. Zheng, G. Tian, S.-C. Huang, D. Feng, A hybrid clustering method for ROI delineation in small-animal dynamic PET images: application to the automatic estimation of FDG input functions, IEEE Transactions on Information Technology in Biomedicine: A Publication of the IEEE Engineering in Medicine and Biology Society 15 (March (2)) (2011) 195-205.

[16] M. Liptrot, K.H. Adams, L. Martiny, L.H. Pinborg, M.N. Lonsdale, N.V. Olsen, S. Holm, C. Svarer, G.M. Knudsen, Cluster analysis in kinetic modelling of the brain: a noninvasive alternative to arterial sampling, NeuroImage 21 (February (2)) (2004) 483-493.

[17] H. Zaidi, M. Diaz-Gomez, A. Boudraa, D.O. Slosman, Fuzzy clustering-based segmented attenuation correction in whole-body PET imaging, Physics in Medicine and Biology 47 (7) (2002) 1143.

[18] J.G. Brankov, N.P. Galatsanos, M.N. Wernick, Segmentation of Dynamic PET or fMRI Images Based on a Similarity Metric, IEEE Transactions on Nuclear Science 50 (October (5)) (2003) 1410-1414.

[19] P. Zanotti-Fregonara, R. Maroy, C. Comtat, S. Jan, V. Gaura, A. Bar-Hen, M.-J. Ribeiro, R. Trébossen, Comparison of 3 methods of automated internal carotid segmentation in human brain PET studies: application to the estimation of arterial input function, Journal of Nuclear Medicine: Official Publication, Society of Nuclear Medicine 50 (March (3)) (2009) 461-467.

[20] R. Maroy, R. Boisgard, C. Comtat, V. Frouin, P. Cathier, E. Duchesnay, F. Dollé, P.E. Nielsen, R. Trébossen, B. Tavitian, Segmentation of rodent whole-body dynamic PET images: 
an unsupervised method based on voxel dynamics, IEEE Transactions on Medical Imaging 27 (March (3)) (2008) 342-354.

[21] R.O. Duda, P.E. Hart, D.G. Stork, Pattern Classification, Wiley-Interscience, New York, 2001, pp. 654.

[22] S. Hapdey, I. Buvat, J.M. Carson, J.M. Carson, J.a. Carrasquillo, M. Whatley, S.L. Bacharach, Searching for alternatives to full kinetic analysis in 18F-FDG PET: an extension of the simplified kinetic analysis method, Journal of Nuclear Medicine: Official Publication, Society of Nuclear Medicine 52 (April (4)) (2011) 634-641.

[23] T.R. DeGrado, M.W. Hanson, T.G. Turkington, D.M. Delong, D.A. Brezinski, J.P. Vallée, L.W. Hedlund, J. Zhang, F. Cobb, M.J.
Sullivan, R.E. Coleman, Estimation of myocardial blood flow for longitudinal studies with $13 \mathrm{~N}$-labeled ammonia and positron emission tomography, Journal of Nuclear Cardiology: Official Publication of the American Society of Nuclear Cardiology 3 (6 (Pt 1)) (1996) 494-507.

[24] P. Zanotti-Fregonara, C.S. Hines, S.S. Zoghbi, J.-S. Liow, Y. Zhang, V.W. Pike, W.C. Drevets, A.G. Mallinger, C.A. Zarate, M. Fujita, R.B. Innis, Population-based input function and image-derived input function for $[(11) C](R)$-rolipram PET imaging: methodology, validation and application to the study of major depressive disorder, NeuroImage (August) (2012). 